\title{
Association between objectively measured sleep timing and habitual dietary intake in university students A cross-sectional study
}

\section{Takahiro Yoshizaki}

Toyo University https://orcid.org/0000-0002-9557-0596

Fumiharu Togo ( $\nabla$ tougou@p.u-tokyo.ac.jp )

The University of Tokyo https://orcid.org/0000-0003-3113-539X

\section{Research}

Keywords: actigraphy, chronotype, social jetlag, circadian rhythm, reward, habitual dietary intake

Posted Date: May 27th, 2020

DOI: https://doi.org/10.21203/rs.3.rs-31090/v1

License: (c) (i) This work is licensed under a Creative Commons Attribution 4.0 International License.

Read Full License 


\section{Abstract}

Background Previous studies suggest that the forced changes in sleep timing and chronotype may be associated with dietary intake. However, no studies have examined associations between objectively measured sleep timing on weekdays and weekends and habitual dietary intake. This study aimed to investigate the associations among university students.

Methods All the 35 healthy university students included in this study were asked to undertake a 7-day wrist activity recording, and to record the bedtimes and wake-up times. All the participants also completed a self-administered questionnaire during the recording period that included demographic characteristics, habitual dietary intakes, and morningness-eveningness. Dietary intakes over the previous 1 month were evaluated using a self-reported dietary history questionnaire (DHQ). The Japanese version of the Morningness-Eveningness Questionnaire (MEQ) was used to measure the self-rated morningnesseveningness. The score for adherence to the Japanese Food Guide Spinning Top (food guide score) was calculated from the results of the DHQ to assess the diet quality. Nocturnal sleep timing on each night was estimated from the recorded wrist activity.

Results Multivariate linear regression showed that a later sleep timing on weekends was significantly $(p<$ $0.05)$ associated with lower intakes of pulses $(\beta=-0.416)$ and dairy products $(\beta=-0.396)$ and a lower food guide score $(\beta=-0.386)$, while the relative sleep timing on weekdays was not $(p>0.05)$. Multivariate linear regression also showed that an earlier relative sleep timing on weekdays was significantly $(p<$ $0.05)$ associated with a lower intake of cereals $(\beta=0.548)$ and a higher intake of sugar and confectioneries $(\beta=-0.461)$, while the sleep timing on weekends was not $(p>0.05)$.

Conclusions The results suggest that later sleep timing on weekends and earlier relative sleep timing on weekdays may be independently associated with unfavorable food intake in young adults. These findings have important implications for the development of novel strategies for improving dietary intakes and for preventing lifestyle-related diseases.

\section{Background}

Current lifestyles in developed countries have been affected and modified by multiple aspects of the $24 / 7$ ( 24 hours a day and 7 days a week) society. In the society, the wake period is expanding into the nighttime due to the added opportunity for activities of daily living during the night or being forced to work at night under artificial light. Such expansions can delay the phase of the central circadian clock (the central clock, which drives the circadian rhythms of behavior and physiology) in the suprachiasmatic nucleus (SCN). The circadian clock is associated with diurnal preference (i.e., chronotype or morningnesseveningness, that is, the degree to which people prefer to be active in the morning or evening). It can also change the phase relationship between the central circadian clock and sleep timing. Previous studies have indicated that the later phase of the circadian clock, greater eveningness, and the changes in the 
phase relationship increase the risk of health problems such as obesity, diabetes, metabolic syndrome, cardiovascular disease, and mortality [1-4].

There is growing evidence supporting the close associations of evening chronotype/preference with unfavorable dietary intakes [5-7], which is a potential factor for weight gain and obesity. For example, previous studies have shown associations of greater evening chronotype/preference with lower intakes of rice, noodles, vegetables, pulses, eggs, and dairy products [8], and with higher intakes of confectioneries $[7,8]$ and sugar-sweetened beverages exist in young adults $[9,10]$. In nurses engaging in day shift, our previous study has indicated that greater eveningness is associated with lower intakes of green/yellow vegetables, white vegetables, fruits, and algae, and with higher intakes of confectioneries/savory snacks, and sugar-sweetened beverages [6].

Recently, an epidemiological study showed associations of sleep timing on free days and the difference between sleep timing on weekdays and weekends with dietary intakes in young adults [11]. Since the circadian clock is a main determinant of bedtime and wake-up time on free days, sleep timing on free days (i.e., chronotype) is associated with the phase of the circadian rhythm in the central clock $[12,13]$. Regarding sleep timing on weekdays, social factors such as school and work start time in students and workers, respectively, also affect the timing [14]. Thus, the results of the previous study suggest that dietary intake may be associated with the forced changes in sleep timing (i.e., changes in phase relationship between the central circadian clock and sleep timing), as well as chronotype. However, to the best of our knowledge, no studies have examined associations of objectively measured sleep timing on weekdays and weekends with habitual dietary intake.

Therefore, in this study, our purpose was to elucidate associations of the objectively measured sleep timing on weekend nights and sleep timing on weekday nights relative to that on weekend nights in reallife situations with habitual dietary intake in university students. To assess sleep timing, we recorded the continuous wrist activity over a week [15]. A previous study has found that sleep timing on Sunday night (i.e., weekday night) relative to that on Saturday night (i.e., weekend night) is positively associated with reward-related reactivity in the brain inhibitory controller (i.e., prefrontal cortex), in mid/late adolescents [16]. Considering together with the indications of the associations between the brain reward system and dietary intake [17], we examined associations of relative sleep timing on weekdays (not the absolute difference between sleep timing on weekdays and weekends) with habitual dietary intake.

\section{Methods}

\section{Participants}


The participants, recruited in this cross-sectional study through flyers and bulletin board poster, were 44 healthy Japanese university students (24 males and 20 females), aged 20-24 years. The sample size required for the multivariate regression analyses was calculated using $\mathrm{G}^{*}$ Power software [18]. The calculation, based on an assumption of 2 independent variables, a priori large effect size (0.35) at an a error level of 0.05 , and a power ( $1-\beta$ error probability) of 0.90 , resulted in a total sample size of 40 . None of the participants took any medications for more than a month before the 7-day recording of wrist activity. Inclusion criteria for participation in the study were: free of unusual events during the 7-day recording period, no sleep disturbance symptoms, and no hospitalization in the past year for eating disorders or serious medical illness. Each participant gave his/her written informed consent to participate, after having been provided with the full description of the test protocol, aim, and prospective effect on their health. All the study procedures were reviewed and approved by the Institutional Review Board of Toyo University (Approval No. TU2016-001).

\section{Study protocol}

All participants completed a self-administered questionnaire during the recording period that included demographic characteristics, morningness-eveningness preference, and habitual food intakes. The questionnaires submitted by the participants were checked for missing data and inconsistencies by a well-trained dietitian. Wrist activity was continuously measured using Actigraph (AMI Ltd., Ardsley, NY), over the 7-day period, including weekdays and weekends under the free-living condition. The Actigraph recording, which began on Wednesday 21:00, was completed on the next Wednesday 21:00. Each participant was asked to wear Actigraph on the wrist of the non-dominant arm and not to remove the device during the recording period except for when taking a bath. To confirm their routine sleep-wake cycle in the free-living condition, we also asked the participants to record their self-rated bedtimes and wake-up times during the recording period in a sleep diary.

\section{Data processing}

Actigraph was programmed to continuously collect activity data in a minute epoch with the zero crossing method, which counted the number of times of zero level crossing in the synthetic acceleration signal during each epoch [19]. When the participants had a non-daily routine during the recording period or had missed the recording because they forgot to wear the device or due to device failure, the participants' data were excluded from the statistical analysis. 
Cole's algorithm [20] was used to score the sleep or wake in each 1-min epoch over the 7-day activity data. To identify the nocturnal sleep duration per day, bedtimes and wake-up times given by the participants in their daily sleep diaries were used to set the interval for calculating the total minutes for sleep epoch per night. Sleep durations on weekdays and weekends were calculated as the mean sleep durations from Sunday to Thursday nights and for Friday and Saturday nights, respectively.

To estimate the nocturnal sleep timing per night, the sleep/wake (0/1) scored data over the 7-day period were firstly processed using a band-pass filtering from 0.9 to $1.1 \mathrm{~Hz}$ for a 24-hour period. Then, the filtered signals were applied to the Hilbert transform to obtain the phase angles of the filtered signal. The timing of sleep (Figure 1) was estimated using the times at \pm pi radians in the transformed data. The sleep timing on weekend nights was calculated as the mean of sleep timing on Friday and Saturday nights, while the sleep timing on weekday nights during the 7-day period was calculated as the mean sleep timing on Sunday, Monday, Tuesday, Wednesday, and Thursday nights. The relative sleep timing on weekday nights during the 7-day period was calculated by subtracting the sleep timing on weekends from the sleep timing on weekdays. A negative value means that the sleep timing on weekday nights is earlier than that on weekend nights.

\section{Self-administered questionnaire}

The questionnaire included demographic characteristics of the participants: age, height, weight, residential status, drinking habits, and smoking habits. Drinking and smoking habits were evaluated on the basis of the National Health and Nutrition Survey definitions [21]: drinking of more than $22 \mathrm{~g}$ of alcohol per day for more than three times per week, and currently smoking and having smoked for more than 6 months, or currently smoking and having smoked a total of 100 cigarettes, respectively. Body mass index (BMI) was calculated on the basis of the self-reported height and weight [weight/height ${ }^{2}$ $\left.\left(\mathrm{kg} / \mathrm{m}^{2}\right)\right]$. The Japanese version of the Horne-Östberg Morningness-Eveningness Questionnaire (MEQ) [22] was used to measure the self-rated diurnal preference, in which lower values indicate greater eveningness. Habitual dietary intakes, except for dietary supplement over the past month were evaluated using a self-reported dietary history questionnaire (DHQ), for which the validity and reproducibility have been reported in a previous study already [23]. Intakes of total energy, protein, fat, carbohydrate, and each food groups were assessed. To examine dietary compositions, each food group intake was adjusted by the total energy intake and sex using the residual method [24-26].

The score for adherence to the Japanese Food Guide Spinning Top (food guide score) was calculated from the results of the DHQ to assess diet quality [27]. Details of the score has been previously shown 
[27]. In brief, the scores (ranging from 0-10 points) for the servings of 7 categories of the Japanese Food Guide Spinning Top, such as grain dishes, vegetable dishes, fish and meat dishes, milk and milk products, fruits, energy intake from confectioneries, and total energy intake were calculated based on the reference amounts. Finally, the scores of all categories were summed to provide the food guide score (ranging from 0-70 points). A higher score represents a greater adherence to the dietary recommendations of the Japanese dietary guidelines.

\section{Statistical analysis}

Of the 44 participants who undertook the 7-day recording, 8 that did not sleep for more than one night for any reasons (e.g., participating in non-habitual events during the nights) during the recording period were excluded from the statistical analyses. Furthermore, a participant with a BMI outlier $\left(43.0 \mathrm{~kg} / \mathrm{m}^{2}\right)$, which was detected by the Smirnov-Grubbs test at a significance level of 0.05 , was excluded. Therefore, 35 participants (21 males and 14 females) were included in our statistical analyses. Pearson's correlation coefficients were used to assess the associations of the sleep timing on weekends and the relative sleep timing on weekdays with demographic characteristics and habitual dietary intakes, and the associations of the sleep durations on weekdays and weekends with habitual dietary intakes. We dichotomized the participants based on the sleep timing on weekends at its median value into early $(n=17)$ and late $(n=$ 18) sleepers, to determine the association between the sleep timing on weekends and the changes in sleep timing over the recording period. Linear mixed-effect model was used to analyze the effects of the group of weekend sleep timing (dummy: early sleepers $=0$, late sleepers $=1$ ), the day of the week (dummy: Wednesday nights $=0$, Thursday/Friday/Saturday/Sunday/Monday/Tuesday nights $=1$ ), and the interaction terms between them on the daily sleep timings. Multivariate linear regressions were performed to explore the independent associations of the sleep timing on weekends and the relative sleep timing on weekdays with habitual food group intakes. Residential status was included as a covariate in the multivariate linear regression analysis (dummy: living alone $=0$, other $=1$ ). To confirm the effects of the excluded participants, we used unpaired $t$-tests and $\chi^{2}$ tests for continuous and categorical variables, respectively, to examine the differences in characteristics between the excluded participants and those included in the main analysis. All statistical analyses were performed using Stata 16 (Stata Corporation, College Station, TX, USA). P values less than 0.05 were considered statistically significant using twotailed tests.

\section{Results}

The mean age, height, weight, $\mathrm{BMl}$, and the proportion of female participants were 21.0 (range, 20-24) years, 166.2 (range, 151.3-184.0) cm, 59.0 (range, 45.5-77.6) kg, 21.3 (range, 17.4-28.0) kg/m², and $40 \%$, respectively. The following characteristics of the excluded participants $(n=9)$ were not significantly different $(p>0.05)$ from those of the included participants in the main analysis: age (mean \pm standard 
deviation [SD]), $21.2 \pm 0.7$ years; height, $163.3 \pm 9.4 \mathrm{~cm}$; weight, $63.1 \pm 27.0 \mathrm{~kg} ; \mathrm{BMl}, 23.2 \pm 7.5 \mathrm{~kg} / \mathrm{m}^{2}$; and the proportion of females, $66.7 \%$.

The associations of the sleep timing on weekends and the relative sleep timing on weekdays with demographic characteristics are shown in Table 1. A later sleep timing on weekends was significantly $(p<$ $0.05)$ associated with a later sleep timing on weekdays $(r=0.706)$, a lower MEQ score $(r=-0.729)$, and an earlier relative sleep timing on weekdays $(r=-0.561)$ (Table 1). An earlier relative sleep timing on weekdays was significantly associated with being less likely to be living alone $(p<0.05)$.

\section{(Please insert Table 1 here)}

The sleep timing over the 7-day recording for the groups of early and late sleepers on weekend are shown in Figure 2. The main effect of the group on the sleep timing (i.e., the difference of the sleep timing for Wednesday night between the groups) and the main effects of the day of the week on the sleep timing were not significant $(p>0.05)$. The interactive effects of the group and the day of the week (group $\times$ day of the week) on the sleep timing were significant $(p<0.05)$ only for Friday and Saturday nights.

Associations between the sleep timing on weekends or the relative sleep timing on weekdays and habitual nutrient or food group intakes are shown in Table 2. A later sleep timing on weekends was significantly $(p<0.05)$ associated with lower intakes of pulses $(r=-0.411)$ and dairy products $(r=$ $-0.410)$. An earlier relative weekend sleep timing on weekdays was significantly $(p<0.05)$ associated with a lower intake of cereals $(r=0.460)$ and a higher intake of sugar and confectioneries $(r=-0359)$. The sleep durations on weekdays and weekends were not associated with any habitual nutrient or food group intakes ( $p>0.05)$, except for the associations between the sleep duration on weekends and intakes of white vegetables $(r=0.347, p<0.05)$ and fats and oils $(r=0.366, p<0.05)$.

\section{(Please insert Table 2 here)}

Regarding the intakes of pulses, dairy products, cereals, and sugar and confectioneries, we examined the independent associations of the sleep timing on weekends and the relative sleep timing on weekdays with the intakes of the food groups (Table 3). Multivariate linear regression showed that a later sleep timing on weekends was significantly $(p<0.05)$ associated with lower intakes of pulses $(\beta=-0.416)$ and 
dairy products $(\beta=-0.396)$ and a lower food guide score $(\beta=-0.386)$, while the relative sleep timing on weekdays was not $(p>0.05)$. Multivariate linear regression also showed that an earlier relative sleep timing on weekdays was significantly $(p<0.05)$ associated with a lower intake of cereals $(\beta=0.548)$ and a higher intake of sugar and confectioneries $(\beta=-0.461)$, while the sleep timing on weekends was not ( $p$ $>0.05$ ).

\section{(Please insert Table 3 here)}

\section{Discussion}

This study aimed to clarify the associations of the objectively measured sleep timing with habitual dietary intake in Japanese university students. We found that sleep timing on weekends was negatively associated with intakes of pulses and dairy products and the food guide score. In addition, we also found that the relative sleep timing on weekdays was positively associated with intake of cereals and significantly negatively associated with intake of sugar and confectioneries. These results suggest that later sleep timing on weekends and earlier relative sleep timing on weekdays may be independently associated with unfavorable food intakes in young adults.

A lower food guide score has been shown to be associated with increased risks of cardiovascular disease and cerebrovascular disease mortality [27]. A previous longitudinal study has indicated that a higher intake of sugar, adjusted for total energy intake, is associated with an increased risk of depression [28]. Considering the fact that young adults tend to have later sleep timing on free days [29], weekend sleep timing and the relative sleep timing on weekdays may be noted for preventing/improving unfavorable food intake in young adults.

Variables of sleep timing including sleep timing on weekends and the relative sleep timing on weekdays correlate with each other, as shown in the results of this study (Figure 2, Table 1), because these timings are associated with the phase of the central circadian clock on free days or diurnal preference for activity timing. For example, on free days, a later phase in the circadian clock or greater evening preference induces later sleep timing (i.e., staying up later at night and waking up later in the morning) [13]. On nonfree days, when there are socially determined schedules including classes or work in the early morning, wake-up time tends to be earlier compared with the time on free days for individuals with a later phase in the circadian clock on free days, or greater evening preference for activity timing. This means that their relative sleep timing on weekdays tend to be earlier compared with those of individuals with an earlier phase in the circadian clock on free days [30]. Partly because of this relationship, it has remained unclear whether sleep timing on weekends and the relative sleep timing on weekdays are independently 
associated with the amounts of habitual food intakes. We have firstly demonstrated that sleep timing on weekends and the relative sleep timing on weekdays may be independently associated with habitual food intakes in young adults.

Previous studies have shown close associations of evening chronotype/preference with unfavorable dietary intake [5-7]. Our results support the association between later sleep timing on weekends (i.e., greater eveningness in chronotype) and a greater unfavorable dietary intake. Regarding the intakes of each food group, an epidemiological study [8] examined the association between the midpoint of sleep on weekdays and habitual dietary intake using the DHQ at a month, which included spring vacation days, among Japanese young adults. The study has shown that later timing of the midpoint of sleep is associated with lower intakes of rice, noodles, vegetables, pulses, eggs, and dairy products; and higher intakes of confectioneries, fats and oils, and meats. Our results, which were obtained after controlling for the relative sleep timing on weekdays, are partly consistent with the results of the previous study.

The observed negative relationship between the relative sleep timing on weekdays and the amount of sugar and confectioneries intake indicates that earlier sleep timing on weekdays compared with that on weekends was associated with a higher intake of sugar and confectioneries, while later sleep timing on weekdays compared with that on weekends was associated with a lower intake of sugar and confectioneries. Although, this association has not been revealed in previous epidemiological studies, associations between the absolute difference between sleep timing on weekdays and weekends (i.e., social jetlag) and food intakes have been examined in undergraduate students and patients with obesityrelated chronic diseases [11,31]. The study among undergraduate students reported that the absolute difference between sleep timing on weekdays and weekends was not associated with the number of the serving of sugar and sweets [11]. In this study, although we found a negative relationship between the relative sleep timing on weekdays and the amount of sugar and confectioneries intake, we could not find a significant association between the absolute difference and the amount of sugar and confectioneries intake $(r=0.2389, p=0.1668)$. The study among patients with obesity-related chronic diseases reported that the presence ( $>1$ hour) of absolute sleep timing difference between weekdays and weekends was significantly associated with the number of serving of sweets [31]. However, it is noted that, in the patients with sleep timing difference, the mean bedtime and wake-up time on weekdays were earlier (100 mins and 180 mins, respectively) than those on weekends. These indicate that the association may be obtained because the group of the presence of sleep timing difference included, primarily, individuals with earlier sleep timing on weekdays compared with that on weekends. Thus, the results of the previous studies do not deny the possibility that later sleep timing on weekdays compared with that on weekends is associated with a lower intake of sugar and confectioneries. 
Although the brain/neuronal mechanisms of the negative linear association of the relative sleep timing on weekdays and the amount of sugar and confectioneries intake are unclear, the results of previous studies on the brain reward system may support the possibility of the association. The acute sleep timing shift, which can be caused by social factors such as school and work start time, and which can change the phase relationship between the central circadian clock and sleep timing, has been reported to be associated with the brain reward system [16]. The previous study has shown that greater weekendweekday advances and delays in sleep timing is associated with decreased and increased medial prefrontal cortex ( $\mathrm{mPFC}$ ) reactivities, respectively, both during reward anticipation and during reward outcome in the mid/late adolescents, while it is not associated with ventral striatum reactivities during reward outcome [16]. Dopamine-mediated reward pathways play a major role in reward-motivated behaviors such as food intakes [32] and involve the MPFC, which acts as a part of the inhibitory controller (i.e., PFC) of the nucleus accumbens [32]. Regarding these associations, for example, transcranial direct current stimulation studies have shown that the active stimulation on the PFC suppresses the craving for sweet foods [33,34]. These results indicate that earlier relative sleep timing on weekdays, and not later relative sleep timing on weekdays, may be associated with an elevated reward-motivated food intakes (e.g., intakes of sugar and confectioneries) because of the reduced regulatory control to reward [16]. In addition, we observed a negative linear relationship $(r=-0.321, p=0.06)$ between the relative sleep timing on weekdays and intake of sugar-sweetened beverages at a trend level (Table 2). The next step would be to examine the brain/neuronal mechanisms of the observed relationship between the relative sleep timing on weekdays and food intakes.

Our results also showed a significant association of earlier weekday sleep timing with a lower intake of cereals. Although, a higher intake of sugar and confectioneries may contribute to a lower amount of intake of cereals because of a significant negative relationship between them $(r=-0.463, p<0.01)$, reasons for the significant association of the relative sleep timing on weekdays with the intake of cereals is unclear. This should also be examined in future studies.

Several limitations in this study should be taken into consideration. First, this cross-sectional study was not able to establish causal association of sleep timing on weekends and the relative sleep timing on weekdays with food group intakes. Longitudinal and interventional studies are required to test whether sleep timing on weekends and the relative sleep timing on weekdays have a certain effect on food intakes and whether the changes in food intakes mediate associations between the sleep timing and lifestylerelated diseases. Second, it is possible that some residual confounding might remain because of unmeasured or non-adjusted factors. Third, we did not assess the circadian phase of the participants. The associations between dietary intake, sleep timing, and measures of circadian phase, such as the acrophase of 24-h melatonin concentration or the dim light melatonin onset need to be further investigated. Finally, the generalization of our results may be limited because the samples consisted of 
only Japanese university students whose dietary quality on average is poorer than that of the average quality in Japanese adults [27]. Studies in other populations may be required.

\section{Conclusions}

Later sleep timing on weekends and earlier sleep timing on weekdays relative to that on weekends may be independently associated with unfavorable food intakes such as less adherence to the dietary recommendations for Japanese, and higher intakes of sugar and confectioneries in young adults. These findings have important implications for the development of novel strategies for improving dietary intakes and for preventing lifestyle-related diseases.

\section{List Of Abbreviations}

BMI: body mass index

DHQ: dietary history questionnaire

Food guide score: adherence to the Japanese Food Guide Spinning Top

MEQ: Morningness-Eveningness Questionnaire

mPFC: medial prefrontal cortex

SCN: suprachiasmatic nucleus

SD, standard deviation

SE, standard error

\section{Declarations}

\section{Ethics approval and consent to participate}

The study was approved by the Institutional Review Board of Toyo University, Tokyo, Japan. The study was also approved by the ethics committee of Toyo University (approval number TU2016-001). All participants provided informed consent.

\section{Consent for publication}

Not applicable 


\section{Availability of data and materials}

We cannot publicly provide individual data due to participants' privacy, according to ethical guidelines in Japan. Additionally, the informed consent we obtained does not include a provision for publicly sharing the data. The datasets used and/or analyzed during the current study are available from the corresponding author on reasonable request.

\section{Competing interests}

The authors have no competing interests.

\section{Funding}

This study was supported by grants from the Japan Society for the Promotion of Science (Grant-in-Aid for Young Scientists (B) 16K16289).

\section{Authors' contributions}

TY and FT contributed to the study design. Material preparation, data collection, and data analysis were performed by TY and FT. TY and FT wrote the first draft of the manuscript, read and approved the final manuscript.

\section{Acknowledgements}

We thank all individuals who participated in this study.

\section{References}

1. Knutson KL, von Schantz M. Associations between chronotype, morbidity and mortality in the UK Biobank cohort. Chronobiol Int. 2018;35:1045-53.

2. Wong PM, Hasler BP, Kamarck TW, Muldoon MF, Manuck SB. Social Jetlag, Chronotype, and Cardiometabolic Risk. J Clin Endocrinol Metab. 2015;100:4612-20.

3. Yu JH, Yun CH, Ahn JH, Suh S, Cho HJ, Lee SK, Yoo HJ, Seo JA, Kim SG, Choi KM, et al. Evening chronotype is associated with metabolic disorders and body composition in middle-aged adults. $\mathrm{J}$ Clin Endocrinol Metab. 2015;100:1494-502. 
4. Togo F, Yoshizaki T, Komatsu T. Association between depressive symptoms and morningnesseveningness, sleep duration and rotating shift work in Japanese nurses. Chronobiol Int. 2017;34:34959.

5. Almoosawi S, Vingeliene S, Gachon F, Voortman T, Palla L, Johnston JD, Van Dam RM, Darimont C, Karagounis LG. Chronotype: Implications for Epidemiologic Studies on Chrono-Nutrition and Cardiometabolic Health. Adv Nutr. 2019;10:30-42.

6. Yoshizaki T, Komatsu T, Tada Y, Hida A, Kawano Y, Togo F. Association of habitual dietary intake with morningness-eveningness and rotating shift work in Japanese female nurses. Chronobiol Int. 2018;35:392-404.

7. Sato-Mito N, Shibata S, Sasaki S, Sato K. Dietary intake is associated with human chronotype as assessed by both morningness-eveningness score and preferred midpoint of sleep in young Japanese women. Int J Food Sci Nutr. 2011;62:525-32.

8. Sato-Mito N, Sasaki S, Murakami K, Okubo H, Takahashi Y, Shibata S, Yamada K, Sato K. The midpoint of sleep is associated with dietary intake and dietary behavior among young Japanese women. Sleep Med. 2011;12:289-94.

9. Li W, Wu M, Yuan F, Zhang H. Sugary beverage consumption mediates the relationship between late chronotype, sleep duration, and weight increase among undergraduates: a cross-sectional study. Environ Health Prev Med. 2018;23:63.

10. Zhang Y, Xiong Y, Dong J, Guo T, Tang X, Zhao Y. Caffeinated Drinks Intake, Late Chronotype, and Increased Body Mass Index among Medical Students in Chongqing, China: A Multiple Mediation Model. Int J Environ Res Public Health. 2018;15.

11. Silva CM, Mota MC, Miranda MT, Paim SL, Waterhouse J, Crispim CA. Chronotype, social jetlag and sleep debt are associated with dietary intake among Brazilian undergraduate students. Chronobiol Int. 2016:1-9.

12. Kitamura S, Hida A, Aritake S, Higuchi S, Enomoto M, Kato M, Vetter C, Roenneberg T, Mishima K. Validity of the Japanese version of the Munich ChronoType Questionnaire. Chronobiol Int. 2014;31:845-50.

13. Crowley SJ, Acebo C, Fallone G, Carskadon MA. Estimating dim light melatonin onset (DLMO) phase in adolescents using summer or school-year sleep/wake schedules. Sleep. 2006;29:1632-41.

14. Wittmann M, Dinich J, Merrow M, Roenneberg T. Social jetlag: misalignment of biological and social time. Chronobiol Int. 2006;23:497-509.

15. Mongrain V, Lavoie S, Selmaoui B, Paquet J, Dumont M. Phase relationships between sleep-wake cycle and underlying circadian rhythms in morningness-eveningness. J Biol Rhythms. 2004;19:24857.

16. Hasler BP, Dahl RE, Holm SM, Jakubcak JL, Ryan ND, Silk JS, Phillips ML, Forbes EE. Weekendweekday advances in sleep timing are associated with altered reward-related brain function in healthy adolescents. Biol Psychol. 2012;91:334-41. 
17. Alonso-Alonso M, Woods SC, Pelchat M, Grigson PS, Stice E, Farooqi S, Khoo CS, Mattes RD, Beauchamp GK. Food reward system: current perspectives and future research needs. Nutr Rev. 2015;73:296-307.

18. Faul F, Erdfelder E, Buchner A, Lang AG. Statistical power analyses using G*Power 3.1: tests for correlation and regression analyses. Behav Res Methods. 2009;41:1149-60.

19. Ancoli-Israel S, Cole R, Alessi C, Chambers M, Moorcroft W, Pollak CP. The role of actigraphy in the study of sleep and circadian rhythms. Sleep. 2003;26:342-92.

20. Cole RJ, Kripke DF, Gruen W, Mullaney DJ, Gillin JC. Automatic sleep/wake identification from wrist activity. Sleep. 1992;15:461-9.

21. Japanese Ministry of Health Labour and Welfare: Results from 2010 National Health and Nutrition Survey. http://www.mhlw.go.jp/bunya/kenkou/eiyou/h22-houkoku.html. Accessed 6 Apr 2020.

22. Ishihara K, Saitoh T, Inoue Y, Miyata Y. Validity of the Japanese version of the MorningnessEveningness Questionnaire. Percept Mot Skills. 1984;59:863-6.

23. Kobayashi S, Murakami K, Sasaki S, Okubo H, Hirota N, Notsu A, Fukui M, Date C. Comparison of relative validity of food group intakes estimated by comprehensive and brief-type self-administered diet history questionnaires against $16 \mathrm{~d}$ dietary records in Japanese adults. Public Health Nutr. 2011;14:1200-11.

24. Willett WC: Nutritional Epidemiology. 2nd ed. New York: Oxford University Press; 1998.

25. Allès B, Baudry J, Méjean C, Touvier M, Péneau S, Hercberg S, Kesse-Guyot E. Comparison of Sociodemographic and Nutritional Characteristics between Self-Reported Vegetarians, Vegans, and Meat-Eaters from the NutriNet-Santé Study. Nutrients. 2017;9.

26. Yatsuya H, Ohwaki A, Tamakoshi K, Wakai K, Koide K, Otsuka R, Mabuchi T, Murata C, Zhang H, Ishikawa $\mathrm{M}$, et al. Reproducibility and validity of a simple checklist-type questionnaire for food intake and dietary behavior. J Epidemiol. 2003;13:235-45.

27. Kurotani K, Akter S, Kashino I, Goto A, Mizoue T, Noda M, Sasazuki S, Sawada N, Tsugane S. Quality of diet and mortality among Japanese men and women: Japan Public Health Center based prospective study. BMJ. 2016;352:i1209.

28. Sanchez-Villegas A, Zazpe I, Santiago S, Perez-Cornago A, Martinez-Gonzalez MA, Lahortiga-Ramos F. Added sugars and sugar-sweetened beverage consumption, dietary carbohydrate index and depression risk in the Seguimiento Universidad de Navarra (SUN) Project. Br J Nutr. 2018;119:211-21.

29. Roenneberg T, Kuehnle T, Pramstaller PP, Ricken J, Havel M, Guth A, Merrow M. A marker for the end of adolescence. Curr Biol. 2004;14:R1038-9.

30. Lewin DS, Wang G, Chen YI, Skora E, Hoehn J, Baylor A, Wang J. Variable School Start Times and Middle School Student's Sleep Health and Academic Performance. J Adolesc Health. 2017;61:20511.

31. Mota MC, Silva CM, Balieiro LCT, Goncalves BF, Fahmy WM, Crispim CA. Association between social jetlag food consumption and meal times in patients with obesity-related chronic diseases. PLoS One. 2019;14:e0212126. 
32. DiLeone RJ, Taylor JR, Picciotto MR. The drive to eat: comparisons and distinctions between mechanisms of food reward and drug addiction. Nat Neurosci. 2012;15:1330-5.

33. Fregni F, Orsati F, Pedrosa W, Fecteau S, Tome FA, Nitsche MA, Mecca T, Macedo EC, Pascual-Leone A, Boggio PS. Transcranial direct current stimulation of the prefrontal cortex modulates the desire for specific foods. Appetite. 2008;51:34-41.

34. Goldman RL, Borckardt JJ, Frohman HA, O'Neil PM, Madan A, Campbell LK, Budak A, George MS. Prefrontal cortex transcranial direct current stimulation (tDCS) temporarily reduces food cravings and increases the self-reported ability to resist food in adults with frequent food craving. Appetite. 2011;56:741-6.

\section{Tables}

Please see the supplementary files section to view the tables.

\section{Figures}

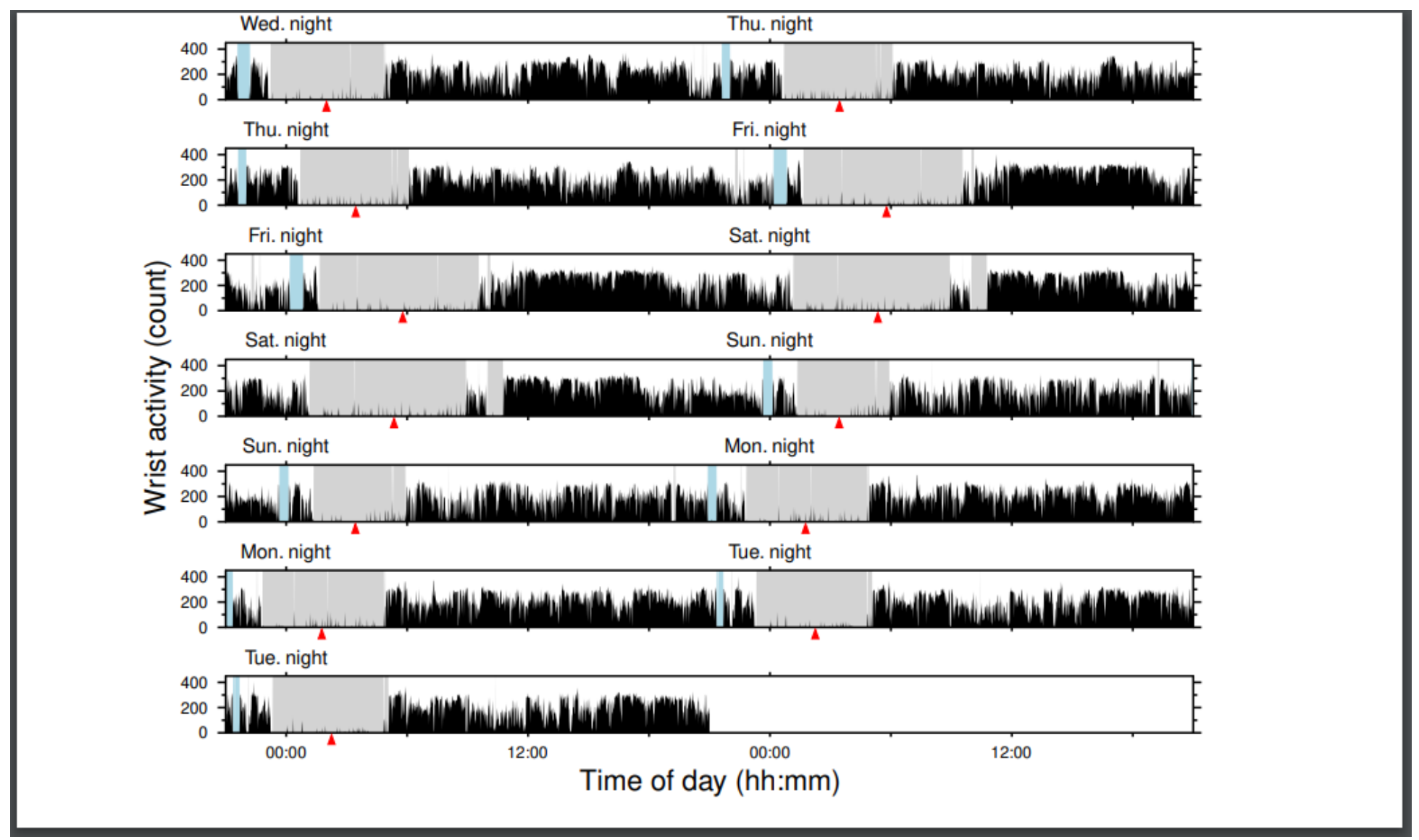

\section{Figure 1}

An example of wrist activity counts and sleep-wake pattern over the 7-day recording. Data are doubleplotted to better visualize changes in sleep timing. Estimated sleep epochs are indicated by the gray zone. 


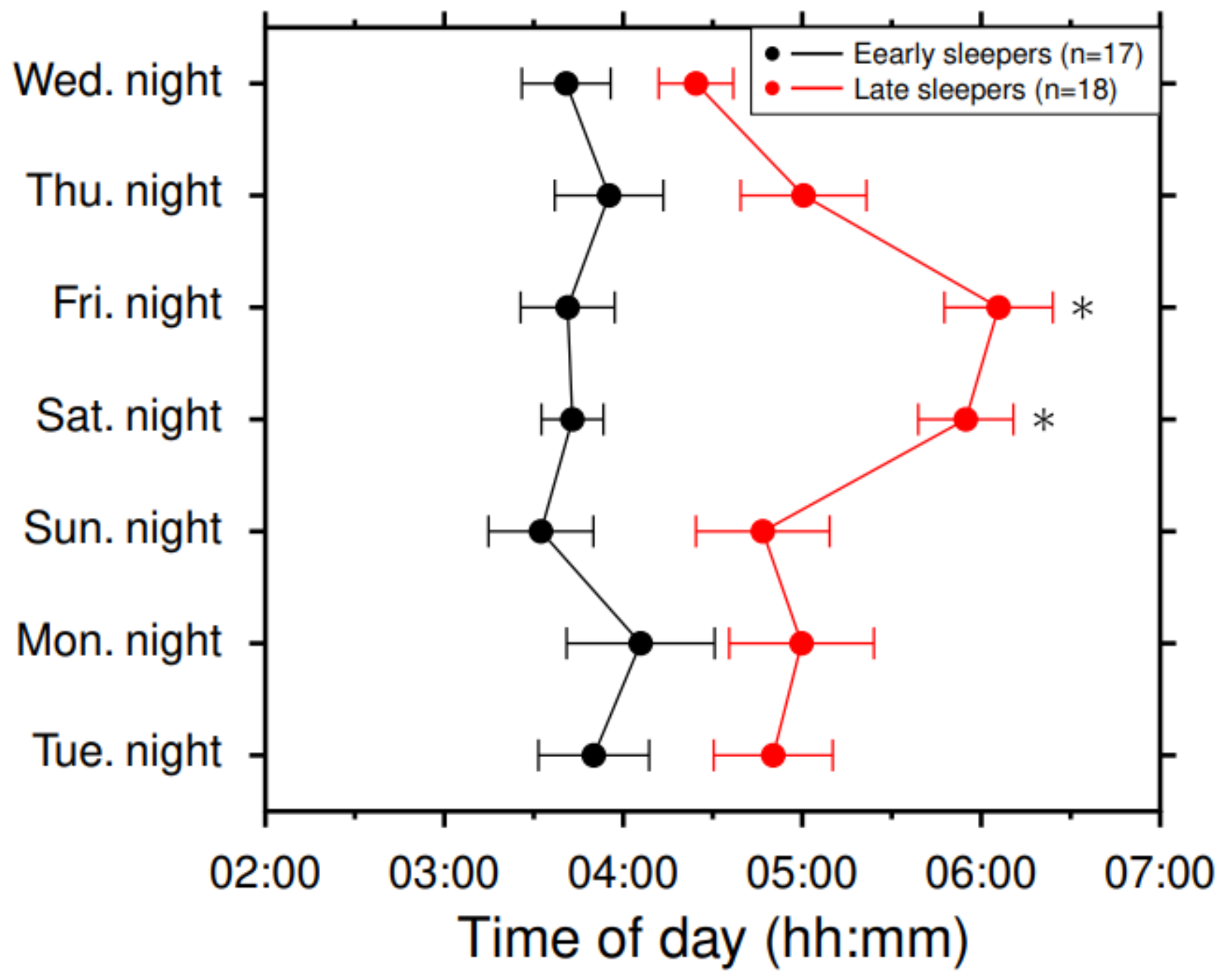

Figure 2

Sleep timing over the 7-day recording for early sleepers and late sleepers on weekend nights. Values are shown as mean \pm SE. * Significant interaction $(p<0.05)$ between the group of weekend sleep timing and the day of the week SE, standard error 

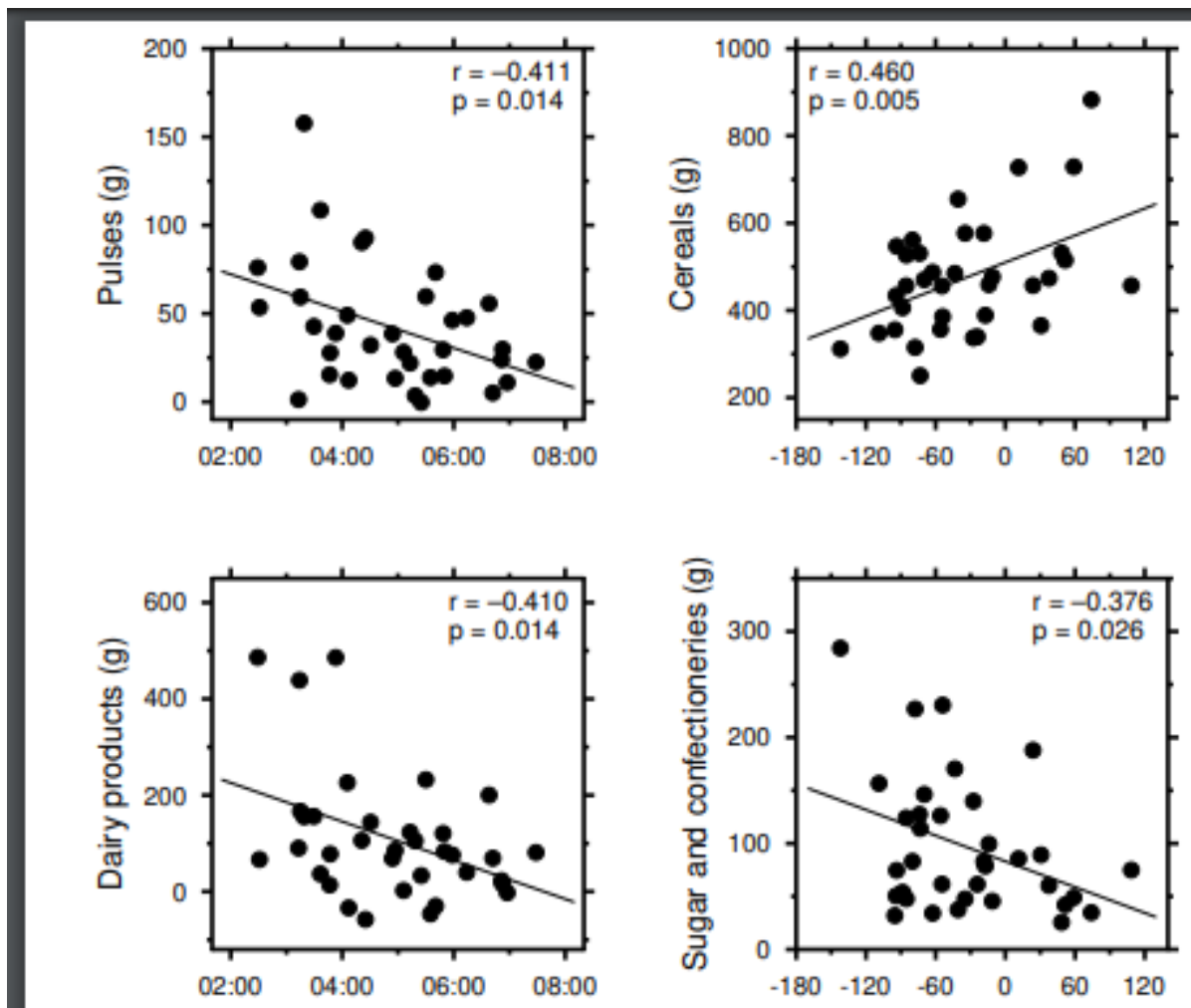

Relative sleep timing on weekdays (mins)

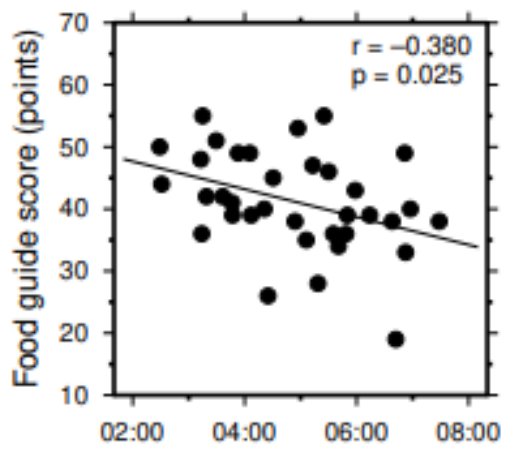

Sleep timing on weekends (hh:mm)

\section{Figure 3}

Relationship between sleep timing and the selected food group intake or the food guide score. Food group intake was adjusted by the total energy intake and sex using the residual method.

\section{Supplementary Files}

This is a list of supplementary files associated with this preprint. Click to download.

- tables20200521.xlsx

- STROBEnutchecklisttablety20200521.docx 